\title{
The natural course of incidentally detected, small, subsolid lung nodules - is follow-up needed beyond current guideline recommendations?
}

\author{
Benedikt H. Heidinger ${ }^{1,2}$, Mario Silva ${ }^{3,4}$, Constance de Margerie-Mellon ${ }^{1}$, Paul A. VanderLaan ${ }^{5}$, \\ Alexander A. Bankier ${ }^{1}$
}

${ }^{1}$ Radiology, Beth Israel Deaconess Medical Center, Harvard Medical School, Boston, MA, USA; ${ }^{2}$ Department of Biomedical Imaging and Imageguided Therapy, Vienna General Hospital, Medical University of Vienna, Vienna, Austria; ${ }^{3}$ Section of Radiology, Unit of Surgical Sciences, Department of Medicine and Surgery (DiMeC), University of Parma, Parma, Italy; ${ }^{4}$ Department of Thoracic Surgery, IRCCS Istituto Nazionale

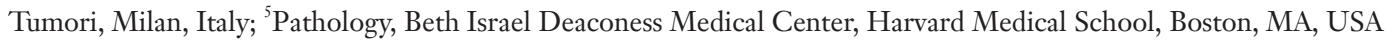

Correspondence to: Benedikt H. Heidinger, MD. Radiology, Beth Israel Deaconess Medical Center, Harvard Medical School, 330 Brookline Ave, Boston, MA 02215, USA. Email: benedikt.heidinger@meduniwien.ac.at.

Provenance: This is an invited article commissioned by the Academic Editor Chenyang Dai, MD, PhD (Department of Thoracic Surgery, Shanghai Pulmonary Hospital, School of Medicine, Tongji University, Shanghai, China).

Comment on: Lee HW, Jin KN, Lee JK, et al. Long-Term Follow-Up of Ground-Glass Nodules After 5 Years of Stability. J Thorac Oncol 2019;14:1370-7.

Submitted Oct 25, 2019. Accepted for publication Nov 04, 2019.

doi: $10.21037 /$ tlcr.2019.11.05

View this article at: http://dx.doi.org/10.21037/tlcr.2019.11.05

Subsolid lung nodules are defined as focal lung lesions on computed tomography (CT) smaller than $3 \mathrm{~cm}$ with a ground glass component $(1,2)$. This category includes nodules with only ground glass attenuation, called "pure ground glass nodules" or "nonsolid nodules", and nodules with both a ground glass and a solid component, called "part-solid nodules" $(1,2)$. Subsolid nodules on CT can represent various pathological entities, from benign to malignant lesions, in particular from the adenocarcinoma spectrum $(3,4)$. However, in an overall perspective, malignant subsolid nodules often grow slowly, have a low metastatic potential and a good prognosis (5-10). Their risk of histological aggressiveness is related to a larger nodule size and both the presence and the size of a solid nodule component, which, in malignant lesions, often represents the invasive tumor component $(4,11)$. To avoid over-management that could potentially cause harm to the patient and an economic burden on health care systems, the risk of malignancy of subsolid nodules is often assessed with longitudinal CT examinations for temporal changes (12). If a nodule is transient, it is assumed to be benign. If a nodule increases in size, develops a new solid component or an existing solid component increases in size, it is suspicious for malignant progression and, as a consequence, calls for action $(1,8)$. The most commonly used guidelines for incidentally detected subsolid nodules, the "Fleischner guidelines", recommend follow-up with CT examinations for at least five years for subsolid nodules with an average diameter of $6 \mathrm{~mm}$ or more that are stable without an increase in nodule size or development of a new solid component (1). For subsolid nodules smaller than $6 \mathrm{~mm}$, follow-up is not recommended. The minimum of five years results from the fact that there is limited data available on the natural course of subsolid nodules that are stable for a prolonged time after discovery (1).

In this context, we read with great interest the recent study by Lee et al. (13). In their retrospective work, the authors investigated the long-term natural course of subsolid lung nodules (13). They included incidentally detected partsolid and nonsolid nodules that were stable with respect to overall nodule size and size of a potential solid component over the five years after detection and followed them for a total of at least 10 years. First, the authors determined the frequency of growth, defined as "interval increase in the long-axis diameter of at least $2 \mathrm{~mm}$ or an increase of long-axis diameter along with the development of a new solid component". 
Then the authors investigated differences in baseline clinical characteristics and radiological features between nodules with and without growth to identify risk factors related to growth.

The authors included 208 subsolid nodules stable over a 5 -year period after detection with a baseline long-axis diameter of $4.7 \mathrm{~mm}$ and about $75 \%$ smaller than $6 \mathrm{~mm}$. The nodules occurred in an Asian population with an age range 28-84 years and smoking history in only about $50 \%$ of patients. One hundred and sixty-two in 208 (78\%) of the nodules were non-solid and 46/208 (22\%) were part-solid. Of included nodules, 27/208 (13.0\%) showed an increase in size on subsequent follow-ups. The median time-togrowth from the baseline examination averaged 8.5 years, with a range of 5.0 to 11.8 years. On multivariate analysis, a history of cancer other than lung cancer, the development of a new solid nodule component and the presence of a bubble lucency were identified as statistically significant risk factors for nodule growth. The authors conclude that even if subsolid nodules are smaller than $6 \mathrm{~mm}$, stable for 5 years, and present in low-risk patients, they should not be ignored, notably when a new solid component appears during follow-up. Although we agree with the importance on understanding the natural course of subsolid nodules, we would like to comment on those findings and the subsequent conclusions.

The authors report on a cohort of lung nodules with an initial median long-axis diameter of $4.7 \mathrm{~mm}$, ranging between 1.7 to $10.0 \mathrm{~mm}$, with the majority of nodules being non-solid nodules. Therefore, most of the nodules included in the study were small. This small size likely results from the fact that larger, subsolid nodules are more likely to represent malignancies and, thus, are more likely to undergo further evaluation via PET-CT or resection early for a final diagnosis. Subsolid nodules smaller than $6 \mathrm{~mm}$ are often transient and have a risk for malignancy of less than $1 \%$ in screening studies $(5-7,14)$. According to current guidelines, only subsolid nodules measuring $6 \mathrm{~mm}$ or more require further follow-up CT (1). Therefore, many of the nodules included in the current study would not require further CT follow-up according to those guidelines. If resected, those subsolid nodules frequently present early cancers from the adenocarcinoma spectrum - particularly adenocarcinoma in situ (AIS) and minimally invasive adenocarcinoma (MIA) frequently showing very slow growth, if any, and rarely contributing to patient mortality $(5-10,15)$. The current study with $13 \%$ of included nodules showing growth after five years of stability supports that adenocarcinomas with subsolid pattern on CT often originate from small nodules with a slow growth and some degree of early stability in size. The authors then state that "it is necessary to follow subsolid nodules for longer periods, even those smaller than $6 \mathrm{~mm}$ ". As subsolid nodules can also be of benign etiology this statement requires caution. Following every small subsolid nodule increases radiation dose for patients, patient anxiety and costs for health care systems (16). Therefore, the decision of following a subsolid smaller than $6 \mathrm{~mm}$ has to be carefully weighed against the cancer risk of an individual patient, among which Asian ethnicity might be a determinant. However, the individual cancer risk is currently is not fully understood and additional data is warranted to better characterize this risk.

Lesion growth was defined by the authors as an increase in long-axis diameter of $2 \mathrm{~mm}$ or more. Two $\mathrm{mm}$ increase is consistent with what is considered growth in the current literature taking technical and observer variability into account $(1,17)$. However, this margin of variability was determined in solid nodules and might be different in subsolid nodules, notably given their often irregular shape and indistinct margins (16). In contrast to current guidelines (18) but similar to the TNM staging approach (19), the authors only present the long-axis diameter in the transverse plane and do not give the average of long- and short-axis diameter. Using the average diameter of long- and short axis results in not perfectly spherical nodules is a more conservative estimate of nodule size and subsequent growth $(18,20)$. Additionally, growth may not be evenly distributed thought the nodule or along its longaxis, and, thus, might not be detectable, when measuring the long-axis only. Finally, maximum growth might not occur in the two dimensions assessed on the transvers CT images but in a craniocaudal direction depicted only in coronal or sagittal CT images (21). It is unclear if nodule measurements for the current study were performed by the authors on transverse CT images only. It would, therefore, be interesting to know how using the average of long- and short-axis as well as three dimensional measurements would have impacted the authors' results.

In univariate analyses, the authors identified female gender and non-smoking as a risk factor, which, however, were not verified in the multivariate analysis. A subset of lung adenocarcinomas, often presenting as subsolid nodules, occurs in patients without the classical risk factors, particularly cigarette smoking. These patients without the classical risk factors are typically Asian women who are non-smokers $(22,23)$. In this particular demographic, the 
prevalence of EGFR-mutated lung cancers is higher than in North American or European cohorts (24). In early studies, EGFR mutations were associated with cancers manifesting as subsolid lung nodules on CT (25), thus, again supporting the notion that there are probably more EGFR-mutated lung adenocarcinomas in the current study. It is, therefore, likely that this subset of adenocarcinomas with a potential enrichment of EGFR-driven lung cancers shows a specific natural course, which might not be representative for any high-risk patient population with an extensive smoking history, limiting broad the recommendations made by the authors until studied in other cohorts.

As a third clinical risk factor, the authors identified a history of previous malignancy other than lung cancer, which remained significant on multivariate analysis. Patients with a history of previous malignancy represent a complex patient population with a higher risk of lung cancer depending on their initial cancer histology, cancer stage and treatment, even if this was a malignancy other than lung cancer (26). Because of the limited amount of data available for this particular population, patients with a history of malignancy are excluded by current guidelines (1). Because of this exclusion by guidelines, follow-up recommendations are at the discretion of the reporting radiologist with a history of previous cancer, substantially influencing their follow-up recommendations (27). Additionally, a study on long-term outcome in lung cancer screening has shown that, despite subsolid lung nodules themselves being indolent, patients do die from lung cancer arising somewhere else in the lung or from cancer other than lung cancer (8). Therefore, future guidelines taking into account the previous history of malignancy could facilitate decision making and potentially improve outcome.

From the assessed radiological features, the development of a new solid component, as well as the presence of a bubbly lesion were associated with growth in both the univariate and the multivariate analyses. In addition to nodule growth, the second change in nodule characteristics that makes a subsolid lung nodule suspicious of malignancy is an increase in nodule density (28). Such an increase in density usually manifests as the development of a new solid component or an increase in size of an already existing solid nodule component. A solid component in the subsolid adenocarcinoma is often the clinical CT correlate of an invasive component on pathology and as consequence the major indicator of malignancy likelihood $(6,11,29-33)$. Indeed, the current 8th TNM staging classification system recommends to use the size of the solid nodule component instead of the overall nodule size, also including the ground glass component for determining an accurate clinical T-stage $(11,19)$. In the presented study by Lee et al., the development of new solid component occurred in the subsolid nodules exhibiting growth in 16/27 (59.3\%) of cases. While this observation seems proof to a causal relation between growth and development of a new solid component, growth as defined by the authors has to be understood.

They defined growth as "an interval increase in the longaxis diameter of at least $2 \mathrm{~mm}$ or an increase of long-axis diameter along with the development of a new solid component", therefore including the development of a new solid component. With this definition, the predictor variable in the presented regression model-development of a new solid component-was part of the outcome variableoverall nodule growth. Therefore, the statistically significant association of the development of a new component with overall nodule growth is to be expected from a methodological point of view and caution is required for its interpretation. In addition to the 16 nodules with growth and development new solid component, a solid nodule component developed in 17/181 (9.4\%) nodules without growth during the follow-up. This underlines findings from prior studies showing the development of solid nodule component while overall size is stable or even decreases in overall size while being of the adenocarcinoma spectrum (28). Those 17 nodules without growth are, however, worrisome for the presence of a malignancy with an invasive component as well. To address this high likelihood of malignancy, the authors should have grouped them separately or by included them into group of nodules with growth, even without an increase in the overall nodule size. Additionally, it would be interesting to learn if an increase in size was related to the development of a new solid component, as this might represent an early sign of invasiveness.

The second radiological feature associated with nodule growth was the presence of a bubble lucency. An illustrative example of such a nodule presenting as bubbly lucency is shown in Figure 1. Such a bubble-like appearance has been previously associated with growth in pure ground glass lesions (10), but a recent meta-analysis showed only limited diagnostic value to discriminate pre-invasive from invasive lesions (34). However, the authors refrain from clearly defining their understanding of bubble lucency. Additionally, this radiological classification is likely to suffer substantially from observer variability, although data is 


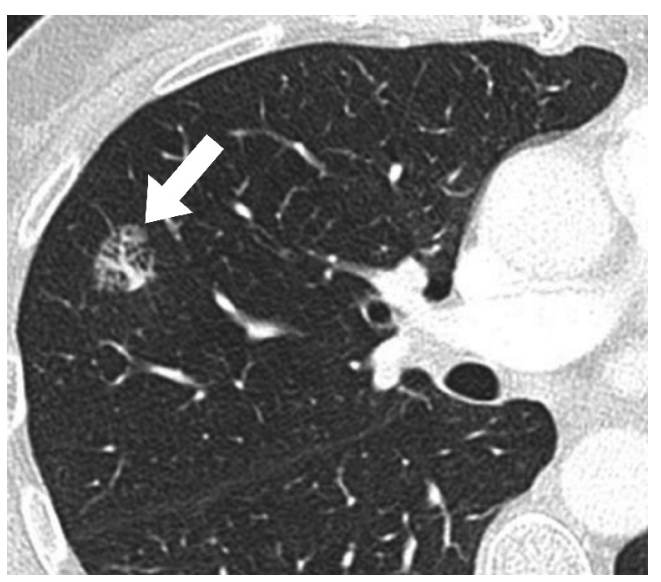

Figure 1 Transverse CT image in lung window settings shows a small subsolid nodule (arrow) in the right upper lobe with tiny, central, hypodense areas, consistent with the manifestation as bubbly lucency.

spares. Also, the number of nodules presenting as bubbly lesion in the current study was small, which limits any generalization.

With the main outcome in the current study being growth, pathological correlation was only available in $3 / 27$ nodules with growth, and in none of the nodules that did not grow. Of those 3 resected nodules, pathology showed one AIS, one MIA, and one invasive adenocarcinoma. Although growth in a lung nodule is considered suspicious for malignancy, it neither proves the nodule to be malignant nor does it predict its clinical course, in particularly in subsolid nodules. It is, however, reassuring that during the available follow-up, no death was reported, thus adding to the evidence of long-term follow-up being a safe strategy in the management of selected subsolid nodules $(8,31,35)$.

In conclusion, to date, information about small lung nodules is limited to solid nodules in the setting of lung cancer screening $(29,36)$. Very little data is available on the natural course of small, subsolid lung nodules that were incidentally detected. The authors of the current study substantially add to fill this gap (13) by showing that a small portion of subsolid lung nodules can still grow after being stable for 5 years. However, it is unclear at that point if and how this finding can impact the broadly recommended clinical management of those nodules. Many of these nodules likely are "indolent" cancers unlikely to ever become of clinical relevance in light of patient age and comorbidities. Unfortunately, it is unlikely that the very long-term studies required to confirm this assumption will ever be performed.

\section{Acknowledgments}

None.

\section{Footnote}

Conflicts of Interest: The authors have no conflicts of interest to declare.

Ethical Statement: The authors are accountable for all aspects of the work in ensuring that questions related to the accuracy or integrity of any part of the work are appropriately investigated and resolved.

\section{References}

1. MacMahon H, Naidich DP, Goo JM, et al. Guidelines for Management of Incidental Pulmonary Nodules Detected on CT Images: From the Fleischner Society 2017. Radiology 2017;284:228-43.

2. Hansell DM, Bankier AA, MacMahon H, et al. Fleischner Society: glossary of terms for thoracic imaging. Radiology 2008;246:697-722.

3. Cho S, Yang H, Kim K, et al. Pathology and prognosis of persistent stable pure ground-glass opacity nodules after surgical resection. Ann Thorac Surg 2013;96:1190-5.

4. Kakinuma R, Noguchi M, Ashizawa K, et al. Natural History of Pulmonary Subsolid Nodules: A Prospective Multicenter Study. J Thorac Oncol 2016;11:1012-28.

5. Henschke CI, Yankelevitz DF, Mirtcheva R, et al. CT screening for lung cancer: frequency and significance of part-solid and nonsolid nodules. AJR Am J Roentgenol 2002;178:1053-7.

6. Henschke CI, Yip R, Smith JP, et al. CT Screening for Lung Cancer: Part-Solid Nodules in Baseline and Annual Repeat Rounds. AJR Am J Roentgenol 2016;207:1176-84.

7. Yankelevitz DF, Yip R, Smith JP, et al. CT Screening for Lung Cancer: Nonsolid Nodules in Baseline and Annual Repeat Rounds. Radiology 2015;277:555-64.

8. Silva M, Prokop M, Jacobs C, et al. Long-Term Active Surveillance of Screening Detected Subsolid Nodules is a Safe Strategy to Reduce Overtreatment. J Thorac Oncol 2018;13:1454-63.

9. Tang EK, Chen CS, Wu CC, et al. Natural History of Persistent Pulmonary Subsolid Nodules: Long-Term Observation of Different Interval Growth. Heart Lung 
Circ 2019;28:1747-54.

10. Takahashi S, Tanaka N, Okimoto T, et al. Long term follow-up for small pure ground-glass nodules: implications of determining an optimum follow-up period and high-resolution CT findings to predict the growth of nodules. Jpn J Radiol 2012;30:206-17.

11. Travis WD, Asamura H, Bankier AA, et al. The IASLC Lung Cancer Staging Project: Proposals for Coding T Categories for Subsolid Nodules and Assessment of Tumor Size in Part-Solid Tumors in the Forthcoming Eighth Edition of the TNM Classification of Lung Cancer. J Thorac Oncol 2016;11:1204-23.

12. Kauczor HU, Baird AM, Blum T, et al. ESR/ERS white paper on lung cancer screening. Eur Respir J 2015;46:28-39.

13. Lee HW, Jin KN, Lee JK, et al. Long-Term Follow-Up of Ground-Glass Nodules After 5 Years of Stability. J Thorac Oncol 2019;14:1370-7.

14. Hammer MM, Palazzo LL, Kong CY, et al. Cancer Risk in Subsolid Nodules in the National Lung Screening Trial. Radiology 2019;293:441-8.

15. Heidinger BH, Anderson KR, Nemec U, et al. Lung Adenocarcinoma Manifesting as Pure Ground-Glass Nodules: Correlating CT Size, Volume, Density, and Roundness with Histopathologic Invasion and Size. J Thorac Oncol 2017;12:1288-98.

16. Hutchinson BD, Moreira AL, Ko JP. Spectrum of Subsolid Pulmonary Nodules and Overdiagnosis. Semin Roentgenol 2017;52:143-55.

17. American College of Radiology. Lung CT Screening Reporting \& Data System (Lung-RADS) 2019. Available online: https://www.acr.org/Clinical-Resources/ Reporting-and-Data-Systems/Lung-Rads. Accessed October 10, 2019.

18. Bankier AA, MacMahon H, Goo JM, et al. Recommendations for Measuring Pulmonary Nodules at CT: A Statement from the Fleischner Society. Radiology 2017;285:584-600.

19. American Joint Comittee on Cancer. AJCC Cancer Staging Manual, 8th edition. New York: Springer; 2018.

20. Heidinger BH, Anderson KR, Moriarty EM, et al. Size Measurement and T-staging of Lung Adenocarcinomas Manifesting as Solid Nodules $\leq 30 \mathrm{~mm}$ on CT: RadiologyPathology Correlation. Acad Radiol 2017;24:851-9.

21. de Margerie-Mellon C, Gill R, Monteira Filho AC, et al. Growth assessment of pulmonary adenocarcinomas manifesting as subsolid nodules on CT: comparison of diameter-based and volume measurements. Acad Radiol
2019. doi: 10.1016/j.acra.2019.09.028.

22. Zhou F, Zhou C. Lung cancer in never smokers-the East Asian experience. Transl Lung Cancer Res 2018;7:450-63.

23. Qiu X, Yuan H, Sima B. Relationship between EGFR mutation and computed tomography characteristics of the lung in patients with lung adenocarcinoma. Thorac Cancer 2019;10:170-4.

24. Zhang YL, Yuan JQ, Wang KF, et al. The prevalence of EGFR mutation in patients with non-small cell lung cancer: a systematic review and meta-analysis. Oncotarget 2016;7:78985-93.

25. Sugano M, Shimizu K, Nakano T, et al. Correlation between computed tomography findings and epidermal growth factor receptor and KRAS gene mutations in patients with pulmonary adenocarcinoma. Oncol Rep 2011;26:1205-11.

26. Toloza EM. Previous tumour as a predictor of risk of developing lung cancer. Thorax 2007;62:373-4.

27. Occhipinti M, Heidinger BH, Pfannenberg C, et al. Managing Incidental Lung Nodules in Patients With a History of Oncologic Disease: A Survey of Thoracic Radiologists. J Thorac Imaging 2017;32:115-20.

28. Scholten ET, de Jong PA, de Hoop B, et al. Towards a close computed tomography monitoring approach for screen detected subsolid pulmonary nodules? Eur Respir J 2015;45:765-73.

29. Walter JE, Heuvelmans MA, de Jong PA, et al. Occurrence and lung cancer probability of new solid nodules at incidence screening with low-dose CT: analysis of data from the randomised, controlled NELSON trial. Lancet Oncol 2016;17:907-16.

30. Yip R, Wolf A, Tam K, et al. Outcomes of lung cancers manifesting as nonsolid nodules. Lung Cancer 2016;97:35-42.

31. Yip R, Yankelevitz DF, Hu M, et al. Lung Cancer Deaths in the National Lung Screening Trial Attributed to Nonsolid Nodules. Radiology 2016;281:589-96.

32. Yip R, Henschke CI, Xu DM, et al. Lung Cancers Manifesting as Part-Solid Nodules in the National Lung Screening Trial. AJR Am J Roentgenol 2017;208:1011-21.

33. Yip R, Li K, Liu L, et al. Controversies on lung cancers manifesting as part-solid nodules. Eur Radiol 2018;28:747-59.

34. Dai J, Yu G, Yu J. Can CT imaging features of groundglass opacity predict invasiveness? A meta-analysis. Thorac Cancer 2018;9:452-8.

35. Pinsky PF, Nath PH, Gierada DS, et al. Short- and longterm lung cancer risk associated with noncalcified nodules 
observed on low-dose CT. Cancer Prev Res (Phila) 2014;7:1179-85.

36. Munden RF, Chiles C, Boiselle PM, et al. Micronodules Detected on Computed Tomography During the

Cite this article as: Heidinger $\mathrm{BH}$, Silva $\mathrm{M}$, de MargerieMellon C, VanderLaan PA, Bankier AA. The natural course of incidentally detected, small, subsolid lung nodules-is followup needed beyond current guideline recommendations? Transl Lung Cancer Res 2019;8(Suppl 4):S412-S417. doi: 10.21037/ tlcr.2019.11.05
National Lung Screening Trial: Prevalence and Relation to Positive Studies and Lung Cancer. J Thorac Oncol 2019;14:1538-46. 